\title{
Pulmonary protection of transcutaneous electrical acupoint stimulation in gynecological laparoscopic surgery: A randomized controlled trial
}

\author{
WEI WEI ${ }^{1,2^{*}}$, WENYA BAI ${ }^{1,3^{*}}$, YANCHAO YANG ${ }^{1}$, YANG LI $^{1}$, \\ XIUFEI TENG ${ }^{1}$, YUXIAO WAN ${ }^{1}$ and JUNCHAO ZHU ${ }^{1}$ \\ ${ }^{1}$ Department of Anesthesiology, Shengjing Hospital of China Medical University; \\ ${ }^{2}$ Department of Anesthesiology, Northeast International Hospital, Shenyang, Liaoning 110004; \\ ${ }^{3}$ Department of Anesthesiology, The First Affiliated Hospital of Kunming Medical University, \\ Kunming,Yunnan 650000, P.R. China
}

Received June 22, 2019; Accepted October 22, 2019

DOI: $10.3892 / \mathrm{etm} .2019 .8245$

\begin{abstract}
Laparoscopy is performed worldwide due to its limited side effects and optimal treatment efficacy. However, it also has adverse effects, including atelectasis and ischemia-reperfusion injury, due to $\mathrm{CO}_{2}$ accumulation during ventilation in a head-down position, which may result in severe disorders and adversely affecting postoperative recovery, prolonging hospitalization. The present study was performed to assess whether transcutaneous electrical acupoint stimulation (TEAS) protects against lung injury occurring during gynecological laparoscopic surgery. Patients were randomly allocated to two groups: Control group (received no stimulation) and TEAS group (patients treated with TEAS on BL13, LI4 and LU5). The mean arterial pressure, heart rate and oxygen saturation were recorded at the time-points of arriving in the operating room $\left(\mathrm{T}_{0}\right)$, immediately prior to induction of the pneumoperitoneum $\left(\mathrm{T}_{1}\right)$, immediately after the end of pneumoperitoneum $\left(\mathrm{T}_{2}\right)$ and on leaving the operating room $\left(\mathrm{T}_{3}\right)$. Arterial blood gas analysis was performed to record
\end{abstract}

Correspondence to: Dr Junchao Zhu, Department of Anesthesiology, Shengjing Hospital of China Medical University, 36 Sanhao Road, Shenyang, Liaoning 110004, P.R. China

E-mail: zhujunchao1@hotmail.com

*Contributed equally

Abbreviations: IRI, ischemia-reperfusion injury; TEAS, transcutaneous electrical acupoint stimulation; TNF- $\alpha$, tumor necrosis factor $\alpha$; IL- $1 \beta$, interleukin $1 \beta$; TENS, transcutaneous electrical nerve stimulation; MAP, mean arterial pressure; HR, heart rate; $\mathrm{SpO}_{2}$, oxygen saturation; $\mathrm{OI}$, oxygen index; $\mathrm{PCO}_{2}$, partial pressure of carbon dioxide

Key words: transcutaneous electric acupoint stimulation, pulmonary protection, gynecologic laparoscopic surgery, post-operative pulmonary complications, oxygen index the $\mathrm{pH}$, determine the partial pressure of carbon dioxide and calculate the oxygenation index (OI) at $\mathrm{T}_{0-3}$. Blood samples were taken from the peripheral vein for determination of the serum concentrations of tumor necrosis factor (TNF)- $\alpha$ and interleukin (IL) $-1 \beta$ at $\mathrm{T}_{0}$ and $\mathrm{T}_{3}$. Post-operative pulmonary complications occurring during the first five days after surgery were also recorded. A total of 100 patients were initially enrolled and 80 patients were analysed. The results indicated that the OI in the control group was significantly lower than that in the TEAS group at the $\mathrm{T}_{2}$ and $\mathrm{T}_{3}$ time-points. The serum concentrations of TNF- $\alpha$ and IL- $1 \beta$ were significantly increased following surgery, while the extent of these increases was lower in the TEAS group compared with that in the control group. The incidence of post-operative pulmonary complications was significantly lower in the TEAS group. It was therefore indicated that TEAS protect against lung injury as a complication of gynecological laparoscopic surgery. The present study was registered at http://www.clinicaltrials.gov prior to enrollment of the patients (no. NCT02850471).

\section{Introduction}

Laparoscopy is performed worldwide due to its limited side effects and optimal healing efficacy compared with traditional surgery. Despite these advantages, laparoscopy has adverse effects, including atelectasis and ischemia-reperfusion injury (IRI), due to $\mathrm{CO}_{2}$ accumulation during ventilation in a head-down position, in particular during gynecological laparoscopic surgery $(1,2)$. These may sometimes result in severe disorders and affect the postoperative recovery, prolong the hospitalization $(3,4)$.

Several different mechanisms account for the adverse effects of pneumoperitoneum and ventilation. Specific trials have been performed proposing that hyperinflation and repetitive tidal recruitment may promote the release of pro-inflammatory mediators, which may lead to lung injury (5). Previous clinical investigations have demonstrated that free reactive oxygen species produced by activation of neutrophils and by the inflammatory response are an essential 
factor for inducing lung IRI (6). A previous trial suggested that impairment of oxygenation contributed to the development of atelectasis. Ventilation is provided due to impairment of lung function, which is accompanied by increased expression of cytokines, including tumor necrosis factor- $\alpha$ (TNF- $\alpha)$ and interleukin $1 \beta$ (IL- $\beta$ ) (7). To date, several trials have demonstrated that mechanical ventilation with lower tidal volumes and positive end-expiratory pressure can prevent pulmonary inflammation and protect against post-operative pulmonary complications $(8,9)$. The underlying mechanism for this could be the reduction of myeloperoxidase and elastase levels in the bronchoalveolar lavage fluid in addition to the inhibition of polymorphonuclear cells activity, which were recruited to the pulmonary compartment $(8,9)$. However, there is currently no consensus regarding the optimal protective ventilation during general anesthesia.

Transcutaneous electrical acupoint stimulation (TEAS) is a complementary and alternative treatment method that combines transcutaneous electrical nerve stimulation (TENS) with traditional acupuncture in order to deliver a specific low-frequency pulse current to the body through the skin. It has been demonstrated to improve the inflammatory response during general anesthesia (10). The present study was performed to test the hypothesis that TEAS protects against lung injury occurring in patients undergoing gynecological laparoscopy under general anesthesia, which is most commonly performed in a head-down position.

\section{Patients and methods}

Study population and design. Ethical approval of this study was granted by the Human Research Ethical Committee of Shengjing Hospital (Shenyang, China; IRB registration no. 2015PS57J). Informed consent was obtained from all participants. The present study was registered at http://www. clinicaltrials.gov prior to enrollment of the patients (registration no. NCT02850471).

A total of 100 patients aged between 18 and 65 years who were scheduled for gynecological laparoscopic surgery under general anesthesia at Shengjing Hospital of China Medical University (Shenyang, China) were included in the present study. The subjects were recruited between March 2015 and July 2015 and were classified according to the American Society of Anesthesiologists physical status classification of I or II (11). The exclusion criteria were as follows: Pre-existing lung or cardiac disease, impaired kidney or liver function, history of bronchial asthma or chronic obstructive pulmonary disease, history of smoking, respiratory infection two weeks prior to enrollment and pre-operative use of a bronchodilator or a steroid.

Randomization and blinding. The patients were randomly assigned either to the TEAS or the control groups using a computer-generated random number sequence. The group assignment was performed in sealed opaque envelopes that were numbered sequentially. The acupuncturist from the Department of Traditional Chinese Medicine of the Shengjing Hospital (Shenyang, China) was informed of the randomization allocation and was only responsible for placing the electrodes and turning on and/or off the stimulator. No communication occurred between him and the investigators of the study. The patients, attending anesthesiologists, surgeons and data collectors were all blinded to the allocation. In order to enhance the reliability of the study, the patients in the control group had electrodes placed at the same acupoints as the patients in the TEAS group. Patients in either of the two groups were told that the acupuncture points would be stimulated by the instrument with a low stimulation frequency so that a person may experience only a mild electric current sensation or feel nothing at all (12).

Study protocol. The patients in the TEAS group were treated with a stimulator (Hwato Electronic Acupuncture Treatment Instrument; model no. SDZ-II; Suzhou Medical Appliances Co., Ltd.) on the Feishu (BL13), Hegu (LI4) and Chize (LU5) acupoints $30 \mathrm{~min}$ prior to the induction of anesthesia. The stimulus wave exhibited an alternate 'dense-disperse' form with the frequency of the disperse wave being $2 \mathrm{~Hz}$ and that of the dense wave being $100 \mathrm{~Hz}$. The optimal intensity was adjusted to maintain a slight twitching of the regional muscle according to the individual maximum tolerance. The stimulus continued until $5 \mathrm{~min}$ prior to the end of surgery. The patients in the control group were also connected to the apparatus, although no electronic stimulation was applied.

Standardized anaesthesia and peri-operative management. All patients fasted overnight and received no pre-medication. Upon arrival in the operating room, standard monitoring, including an electrocardiogram lead II, non-invasive arterial blood pressure and pulse oximetry to measure the oxygen saturation $\left(\mathrm{SpO}_{2}\right)$ were applied. Furthermore, venous access was established using a 18-gauge cannula on the dorsum of the hand, connected to a T-connector. Transfusion was performed with lactated Ringer's solution at a rate of 30 drops per min. General anesthesia was induced with Sufentanil citrate injection $(0.2 \mu \mathrm{g} / \mathrm{kg})$ and Etomidate fat emulsion injection $(2.5 \mathrm{mg} / \mathrm{kg})$. Cisatracurium Besylate injection $(0.2 \mathrm{mg} / \mathrm{kg})$ was used to facilitate intubation. After adequate jaw relaxation was attained, tracheal intubation was performed and each patient was mechanically ventilated with the tidal volume and ventilation rate adjusted to maintain the pressure of end-tidal carbon dioxide at $35-45 \mathrm{mmHg}$ and the $\mathrm{SpO}_{2}$ at $\geq 92 \%$. Maintenance of anesthesia was achieved by administration of sevoflurane in an air-oxygen mixture at a fresh gas flow rate of $21 / \mathrm{min}$. The bispectral index was maintained between 40 and 55 during the operation by adjusting the flow rate of sevoflurane. If the MAP was lower than the fundamental value of $30 \%$, the patients were administered $6 \mathrm{mg}$ hydrochloride ephedrine. If the MAP was $>120 \%$, urapidil hydrochloride $(0.10-0.15 \mathrm{mg} / \mathrm{kg})$ was administered. Atropine sulfate $(0.2 \mathrm{mg})$ was administered if the heart rate $(\mathrm{HR})$ was $<50$ beats per min. One anaesthesiologist who was blinded to the grouping performed the anaesthesia. The same group of experienced surgeons performed all of the surgeries. Following extubation, the patients were transferred to the post-anaesthesia care unit for continuous monitoring.

Hemodynamic index and blood gas analysis index. The mean arterial pressure (MAP), $\mathrm{HR}$ and $\mathrm{SpO}_{2}$ were recorded at the following time-points: Arrival at the operating room $\left(\mathrm{T}_{0}\right)$, immediately prior to induction of the pneumoperitoneum $\left(\mathrm{T}_{1}\right)$, 
Table I. Comparison of patient characteristics.

\begin{tabular}{|c|c|c|c|}
\hline Item & TEAS group $(n=41)$ & Control group $(n=39)$ & P-value \\
\hline Age (years) & $44.7 \pm 10.8$ & $47.3 \pm 11.0$ & 0.303 \\
\hline Body weight (kg) & $55.1 \pm 5.4$ & $57.0 \pm 6.1$ & 0.148 \\
\hline Body height (cm) & $160.2 \pm 3.9$ & $159.9 \pm 3.8$ & 0.792 \\
\hline Duration of anaesthesia (min) & $157.2 \pm 41.2$ & $168.1 \pm 51.6$ & 0.300 \\
\hline Duration of surgery (min) & $140.1 \pm 39.4$ & $149.3 \pm 49.3$ & 0.364 \\
\hline \multicolumn{4}{|l|}{ Surgery type } \\
\hline Total hysterectomy & $9(22.0)$ & $9(23.1)$ & 0.904 \\
\hline Subtotal hysterectomy & $3(7.3)$ & $1(2.6)$ & 0.616 \\
\hline Total hysterectomy and bilateral appendectomy & $3(7.3)$ & $4(10.3)$ & 0.709 \\
\hline Myomectomy & $12(29.3)$ & $7(17.9)$ & 0.234 \\
\hline Oophorocystectomy & $12(29.3)$ & $13(33.3)$ & 0.695 \\
\hline Radical hysterectomy & $0(0)$ & $1(2.6)$ & 0.488 \\
\hline Radical hysterectomy and pelvic lymphadenectomy & $2(4.9)$ & $4(10.3)$ & 0.426 \\
\hline
\end{tabular}

Values are expressed as the mean \pm standard deviation or $\mathrm{n}(\%)$. TEAS, transcutaneous electric acupoint stimulation.

immediately after the end of pneumoperitoneum $\left(\mathrm{T}_{2}\right)$ and on leaving the operating room $\left(\mathrm{T}_{3}\right)$. Arterial blood samples were collected from the radial artery or dorsal artery of the foot for blood gas analysis to record $\mathrm{pH}$, determine the partial pressure of carbon dioxide $\left(\mathrm{PCO}_{2}\right)$ and calculate the oxygenation index (OI) at $\mathrm{T}_{0-3}$.

Serum concentrations of TNF- $\alpha$ and $I L-1 \beta$. Blood samples were taken from the peripheral vein for determination of serum concentrations of tumor necrosis factor $\alpha(\mathrm{TNF}-\alpha)$ and interleukin $1 \beta$ (IL-1 $\beta$ ) at $\mathrm{T}_{0}$ and $\mathrm{T}_{3}$. Peripheral venous blood samples were collected into pre-cooled anti-coagulant tubes and subsequently centrifuged at 3,000 x g for $10 \mathrm{~min}$ at the temperature of $4^{\circ} \mathrm{C}$. The plasma was transferred into polyethylene tubes and stored at $-80^{\circ} \mathrm{C}$. The plasma concentration levels of TNF- $\alpha$ (cat. no. 20150220), IL-1 $\beta$ (cat. no. 20150208) were measured using ELISA kits (Shanghai Kmaels Biological Technology Co., Ltd.; http://www.kmsbiotech.com) according to the manufacturer's protocols, following which absorbance was read at wavelength of $450 \mathrm{~nm}$ using the multifunctional microplate reader (Thermo Fisher Scientific, Inc.).

Postoperative pulmonary complications. Post-operative pulmonary complications that occurred during the first 5 days after surgery were also documented. The complications included hypoxemia, bronchospasm, suspected pulmonary infection, pulmonary infiltrate, aspiration pneumonitis, atelectasis, pleural effusion, pulmonary edema and pneumothorax. Hypoxaemia was defined as $\mathrm{SpO}_{2}<90 \%$ during $\geq 10 \%$ of the total recording time (13). Bronchospasm was defined as a sudden constriction of the muscles of bronchioles. It is a symptom of pulmonary conditions, namely asthma, chronic bronchitis, anaphylaxis, dyspnea, anoxia, asthma and asphyxia. Pneumonia was defined as a new development of pulmonary infiltration detected by chest X-ray. It is characterized by acute respiratory symptoms (cough, fever, sputum production or pleuritic chest pain) and elevated inflammatory markers. This condition requires antibiotic treatment $(14,15)$. Atelectasis was defined as large-size atelectasis of lobar involvement detected by chest X-ray. Pleural effusion was defined as an abnormal accumulation of fluid in the pleural cavity, which is $>0.1-0.2 \mathrm{ml} / \mathrm{kg}$ body weight (16). Pulmonary edema was defined as radiologically diagnosed pulmonary interstitial fluid accumulation. Pneumothorax was defined as an abnormal accumulation of gas within the pleural space, which is usually confirmed by imaging techniques. For instance, the chest radiographs of pneumothorax include a white visceral pleural line separating the lung from the chest wall, accompanied by loss of standard lung markings located in the periphery of this white line (17).

Sample size. The sample size calculation was based on the rate of change of the levels of the IL- $1 \beta$ cytokine prior to and immediately after the surgery $(18,19)$. A preliminary study demonstrated that 34 patients in each group were required for a $10 \%$ difference of IL- $1 \beta$ between the groups immediately after the surgery, assuming a two-sided Type I error $(\alpha)$ of 0.05 and a power of $90 \%$. Potential loss was expected during follow-up or due to dropout and therefore, the sample size was increased to 100 patients.

Statistical analysis. SPSS 19.0 statistical software (IBM Corp.) was employed for data analysis. The normality of distribution was assessed using Kolmogorov-Smirnov test. Data that followed a normal distribution were expressed as the mean \pm standard deviation or $\mathrm{n}(\%)$. All continuous variables were compared using an independent Student's t-test or the Mann-Whitney U-test. The repeated measurement values were compared at each time-point using repeated analysis of variance followed by Bonferroni correction for multiple comparisons. Categorical variables were expressed as n (\%) and were compared using the chi-squared test or Fisher's exact test as appropriate. $\mathrm{P}<0.05$ was considered to indicate a significant difference. 


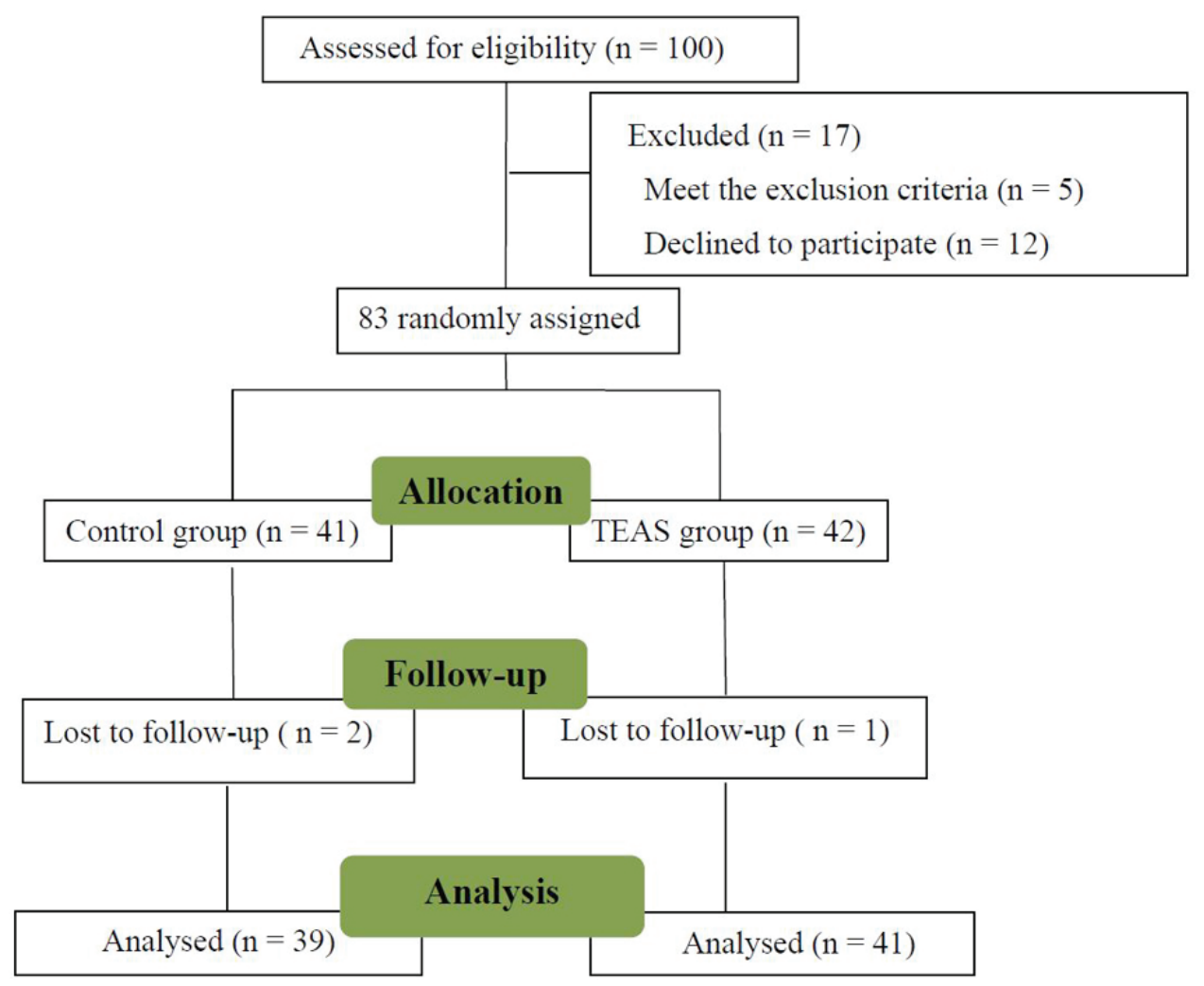

Figure 1. Flowchart depicting the movement of patients throughout the present study. TEAS, transcutaneous electrical acupoint stimulation.

\section{Results}

Participant Enrollment. Between March 2015 and July 2015, 100 patients were enrolled at Shengjing Hospital (Shenyang, China). A flow chart depicting the patient enrolment and their movement throughout the study is provided in Fig. 1. A total of 41 and 39 patients were randomly allocated to the TEAS and the control group, respectively. A total of 20 patients were excluded from the analysis due to consent withdrawal $(n=12)$, satisfaction of exclusion criteria $(n=5)$ and due to loss to follow-up $(n=3)$. The patient characteristics and the type and duration of their procedures (including the surgical type) were not significantly different between the two groups $(\mathrm{P}>0.05$, Table I).

Comparison of MAP, $\mathrm{HR}, \mathrm{SpO}_{2}$, blood $\mathrm{pH}, \mathrm{PCO}_{2}$ and $\mathrm{OI}$. The MAP, $\mathrm{HR}, \mathrm{SpO}_{2}$, blood $\mathrm{pH}, \mathrm{PCO}_{2}$ and OI were compared at various time-points (Table II). No significant differences were noted between the MAP, HR and $\mathrm{SpO}_{2}$ in these two groups $(\mathrm{P}>0.05)$. Blood gas analysis demonstrated no significant differences in terms of $\mathrm{pH}$ and $\mathrm{PCO}_{2}$. However, the $\mathrm{OI}$ in the control group was significantly lower than that in the TEAS group $(\mathrm{P}<0.05$, Table II) at the timepoints of $\mathrm{T}_{2}$ and $\mathrm{T}_{3}$.

Serum concentrations of TNF- $\alpha$ and IL-1 $\beta$. The serum concentrations of TNF- $\alpha$ and IL-1 $\beta$ were significantly increased following surgery $(\mathrm{P}<0.05$, Fig. 2). The extent of the increase of the serum concentration of TNF- $\alpha$ and IL-1 $\beta$ in the TEAS group was significantly lower than that in the control group $(\mathrm{P}<0.05)$.
Postoperative pulmonary complications. Postoperative pulmonary complications were reported in 3 out of 41 patients (7.3\%) in the TEAS group compared with 10 out of 39 patients (25.6\%) in the control group $(\mathrm{P}<0.05$; Table III). The results indicated that hypoxemia was the most common complication following laparoscopy, where the incidence was lower in the TEAS group compared with that in the Control group $(\mathrm{P}<0.05)$. But no significant differences were noted between the two groups in the other items.

\section{Discussion}

Gynecological laparoscopic surgery is frequently performed in a head-down position to facilitate laparoscopic surgical manipulation in which carbon dioxide inflation of the pneumoperitoneum is induced $(1,8)$, which was also performed in the present study. As a result of the head-down body position, mechanical ventilation and abdominal pressure are increased, which impairs the respiratory function. This increases the risk of post-operative pulmonary complications, including atelectasis formation and IRI $(5,8,20)$. Atelectasis and ischemia and reperfusion may trigger several different physiological responses, including pulmonary edema, as well as lymphocyte and neutrophil infiltration, which activate the immune system and result in a hypercoagulable state. IRI is an inflammatory process that occurs in the organs during anoxia (20).

The pathophysiology of atelectasis and IRI in the lungs is complex and multifactorial. Previous studies have indicated that a variety of inflammatory mediators are responsible for inducing cell damage by regulating vascular permeability and leukocyte activity (6). Furthermore, it was demonstrated that 
Table II. Comparison of the variables measured at different time-points.

\begin{tabular}{|c|c|c|}
\hline Parameters & $\begin{array}{l}\text { TEAS group } \\
\quad(n=41)\end{array}$ & $\begin{array}{l}\text { Control group } \\
\qquad(\mathrm{n}=39)\end{array}$ \\
\hline \multicolumn{3}{|c|}{ MAP (mmHg) } \\
\hline $\mathrm{T}_{0}$ & $89.7 \pm 7.9$ & $90.7 \pm 10.6$ \\
\hline $\mathrm{T}_{1}$ & $86.2 \pm 9.6$ & $86.2 \pm 10.8$ \\
\hline $\mathrm{T}_{2}$ & $83.1 \pm 10.9$ & $86.2 \pm 10.7$ \\
\hline $\mathrm{T}_{3}$ & $86.2 \pm 10.1$ & $86.1 \pm 10.1$ \\
\hline \multicolumn{3}{|c|}{ HR (beats/min) } \\
\hline $\mathrm{T}_{0}$ & $76.1 \pm 10.7$ & $74.7 \pm 12.7$ \\
\hline $\mathrm{T}_{1}$ & $74.2 \pm 10.8$ & $69.4 \pm 11.7$ \\
\hline $\mathrm{T}_{2}$ & $69.8 \pm 9.7$ & $70.1 \pm 11.2$ \\
\hline $\mathrm{T}_{3}$ & $73.7 \pm 10.5$ & $78.3 \pm 9.8$ \\
\hline \multicolumn{3}{|l|}{$\mathrm{SpO}_{2}$} \\
\hline $\mathrm{T}_{0}$ & $99.4 \pm 0.6$ & $99.5 \pm 0.5$ \\
\hline $\mathrm{T}_{1}$ & $99.6 \pm 0.6$ & $99.5 \pm 0.7$ \\
\hline $\mathrm{T}_{2}$ & $99.6 \pm 0.5$ & $99.5 \pm 0.9$ \\
\hline $\mathrm{T}_{3}$ & $99.7 \pm 0.8$ & $99.6 \pm 0.9$ \\
\hline \multicolumn{3}{|l|}{$\mathrm{pH}$} \\
\hline $\mathrm{T}_{0}$ & $7.4 \pm 0.04$ & $7.4 \pm 0.06$ \\
\hline $\mathrm{T}_{1}$ & $7.3 \pm 0.06$ & $7.3 \pm 0.04$ \\
\hline $\mathrm{T}_{2}$ & $7.3 \pm 0.06$ & $7.3 \pm 0.03$ \\
\hline $\mathrm{T}_{3}$ & $7.4 \pm 0.03$ & $7.4 \pm 0.04$ \\
\hline \multicolumn{3}{|c|}{$\mathrm{PCO}_{2}(\mathrm{mmHg})$} \\
\hline $\mathrm{T}_{0}$ & $42.8 \pm 6.1$ & $44.4 \pm 6.8$ \\
\hline $\mathrm{T}_{1}$ & $44.4 \pm 8.8$ & $45.0 \pm 8.1$ \\
\hline $\mathrm{T}_{2}$ & $42.1 \pm 8.1$ & $45.2 \pm 7.6$ \\
\hline $\mathrm{T}_{3}$ & $39.5 \pm 4.9$ & $39.9 \pm 5.9$ \\
\hline \multicolumn{3}{|l|}{ OI } \\
\hline $\mathrm{T}_{0}$ & $425.6 \pm 21.2$ & $423.2 \pm 22.0$ \\
\hline $\mathrm{T}_{1}$ & $450.5 \pm 30.0$ & $442.3 \pm 28.9$ \\
\hline $\mathrm{T}_{2}$ & $491.6 \pm 37.8^{\mathrm{a}}$ & $460.1 \pm 39.3$ \\
\hline $\mathrm{T}_{3}$ & $423.7 \pm 25.3^{\mathrm{a}}$ & $395.0 \pm 31.5$ \\
\hline
\end{tabular}

Values are expressed as the mean \pm standard deviation. ${ }^{\mathrm{a}} \mathrm{P}<0.05$ compared with the patients in the Control group. TEAS, transcutaneous electric acupoint stimulation; MAP, mean arterial pressure; HR, heart rate; $\mathrm{OI}$, oxygen index; $\mathrm{PCO}_{2}$, partial pressure of carbon dioxide; $\mathrm{T}_{0}$, time-point of arriving at the operating room; $\mathrm{T}_{1}$, time-point immediately prior to inducing the pneumoperitoneum; $\mathrm{T}_{2}$, time-point immediately after the end of pneumoperitoneum; $\mathrm{T}_{3}$, on leaving the operating room.

cerebral ischemia and reperfusion was due to inflammation partly caused by cerebral ischemia (21). Cytokine signaling is a unique way to respond to particular cellular stimuli. Pulmonary injury caused by atelectasis and IRI is a particular consequence of inflammatory reaction. During this reaction, the inflammatory effector cells, including neutrophils, are activated and facilitate the release of specific cytokines in response to specific chemotactic signals (22). A previous study showed that IL- $1 \beta$ and TNF- $\alpha$ contributed to the eradication of the infection by engaging immune and non-immune cells (23).

\section{Comparison of the serum concentration of TNF- $\alpha$}

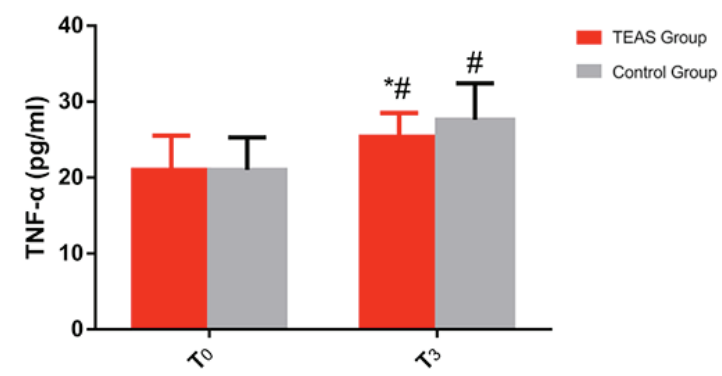

Comparison of the serum concentration of IL-1 $\beta$

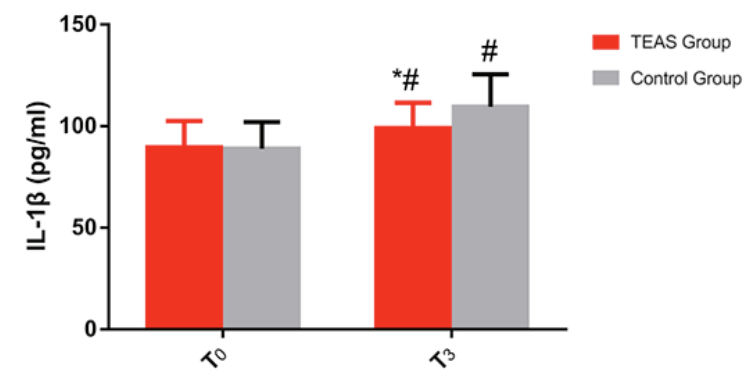

Figure 2. Comparison of the serum concentrations of TNF- $\alpha$ and IL-1 $\beta$. ${ }^{\text {" }} \mathrm{P}<0.05$ compared with the time-point of $\mathrm{T}_{0}$. $\mathrm{P}<0.05$ compared with patients in the Control group. TEAS, transcutaneous electric acupoint stimulation; TNF- $\alpha$, tumor necrosis factor- $\alpha$; IL-1 $\beta$, interleukin- $1 \beta ; \mathrm{T}_{0}$, time-point of arriving at the operating room; $\mathrm{T}_{3}$, leaving the operating room.

Table III. Post-operative pulmonary complications in the first five days after surgery.

\begin{tabular}{llc}
\hline Item & $\begin{array}{c}\text { TEAS group } \\
(\mathrm{n}=41)\end{array}$ & $\begin{array}{c}\text { Control group } \\
(\mathrm{n}=39)\end{array}$ \\
\hline Total & $3(7.3)^{\mathrm{a}}$ & $10(25.6)$ \\
Hypoxaemia & $0(0)^{\mathrm{a}}$ & $4(10.3)$ \\
Bronchospasm & $0(0)$ & $0(0)$ \\
Suspected pulmonary infection & $1(2.4)$ & $1(2.6)$ \\
Pulmonary infiltrate & $0(0)$ & $2(5.1)$ \\
Aspiration pneumonitis & $0(0)$ & $0(0)$ \\
Atelectasis & $1(2.4)$ & $1(2.6)$ \\
Pleural effusion & $1(2.4)$ & $2(5.1)$ \\
Pulmonary edema & $0(0)$ & $0(0)$ \\
Pneumothorax & $0(0)$ & $0(0)$ \\
\hline
\end{tabular}

Values are expressed as $\mathrm{n}(\%) .{ }^{\mathrm{a}} \mathrm{P}<0.05$ compared with the patients in the Control group. TEAS, transcutaneous electric acupoint stimulation.

TNF- $\alpha$ and IL- $1 \beta$ are typical cytokines released in the early phase of the inflammatory reaction. Furthermore, TNF- $\alpha$ and IL-1 $\beta$ are able to activate the cytokine signaling network and induce cellular apoptosis (24-27). During cerebral ischemia, the expression levels of TNF- $\alpha$ and IL- $1 \beta$ are significantly increased, which may further exacerbate brain injury (28). A specific signaling pathway is activated following nuclear translocation of NF- $\mathrm{KB}$ during IRI. This signaling pathway may further activate downstream associated pro-inflammatory cytokines, including TNF- $\alpha$ and IL-1 $\beta$, which in turn induce 
inflammatory reactions $(29,30)$. Cannizzaro et al (31) demonstrated that atelectasis was associated with the production of TNF- $\alpha$, whereas neutrophil infiltration was associated with changes in IL-1 $\beta$ expression levels. Furthermore, secretion of TNF- $\alpha$ and IL-1 $\beta$ has been noted in pulmonary edema (32-34). Atelectasis and IRI may cause damage to pulmonary cells. It was further indicated that TNF- $\alpha$ and IL- $1 \beta$ levels were increased following surgery, suggesting that lung atelectasis and IRI may occur during surgery.

TEAS is a unique treatment method, which delivers a specific low-frequency pulse current to the body through the skin at specific acupoints via TENS. TEAS induce less pain and injury and may cause a lower incidence of infection compared with traditional electroacupuncture. In addition, it exhibits optimal patient tolerability. The response efficacy critically depends on the stimulation parameters. According to the theory of Traditional Chinese Medicine, the Feishu acupoint (BL13) is mainly used to treat cough, asthma, chest fullness, spitting blood and other signs associated with pulmonary infections. Furthermore, acupuncture at BL13 may improve pulmonary function and reduce lung tissue and cell inflammation (35-38). Acupuncture at the Hegu acupoint (LI4) is considered to exert an analgesic and sedative effect and is a key acupuncture point for anesthesia (39). Previous studies have demonstrated that acupuncture at LI4 may effectively improve the remodeling of the cough reflex in patients with tracheotomy following cerebral hemorrhage and immunosuppression in patients undergoing pneumonectomy $(40,41)$. The Chize acupoints (LU5) belong to the Lung Meridian of Hand-Taiyin and may be used to treat cough due to their potential analgesic effects (42).

TEAS exert various effects, including inhibition of apoptosis, anti-inflammatory effects and cell protection $(43,44)$. Previous studies on IRI indicated that electroacupuncture is able to protect the brain against injury through inhibition of apoptosis, reduction of oxidative stress and inhibition of the inflammatory response $(45,46)$. Animal and human studies have suggested that acupuncture may be used to treat dyspnea by stimulating the release of endogenous opioid compounds and by regulating the limbic system $(47,48)$. Previous studies reported that electroacupuncture exerted anti-inflammatory effects and that it was able to cause a decrease in the expression levels of TNF- $\alpha$ and IL-1 $\beta$ (49-51). In the present study, TEAS decreased the expression levels of TNF- $\alpha$ and IL-1 $\beta$ and improved the inflammatory response.

The OI is an important indicator of the oxygenation status of the lung with a higher value indicating better oxygenation of the lung. The present study suggested that the OI in the control group was significantly lower than that in the TEAS group at the different time-points of $\mathrm{T}_{2}$ and $\mathrm{T}_{3}$. The serum concentrations of TNF- $\alpha$ and IL- $1 \beta$ were significantly increased following surgery, which indicates inflammatory reactions during laparoscopic surgery. In addition, the serum concentrations of TNF- $\alpha$ and IL- $1 \beta$ were increased in the TEAS group to a lower extent than those in the control group, which is consistent with the conclusion that TEAS may inhibit the inflammatory response caused by laparoscopic surgery and protect against lung injury, as well as reduce the incidence of pulmonary complications. However, further studies are required to explore the mechanisms of the protective effect of TEAS against pulmonary inflammation.
A significant limitation of the present study was that only relatively healthy female subjects were included in the study protocol (without cardiovascular and/or cerebrovascular diseases and/or pulmonary disease). Therefore, it remains to be determined whether the results may be generalized to patients with pulmonary diseases or to patients who are critically ill. However, the present study is the first to demonstrate that TEAS may be beneficial in reducing the pulmonary injury caused by gynecological laparoscopic surgery.

In conclusion, ventilation-associated lung injury in the pneumoperitoneum, which may be attributed to inflammatory injury, induction of atelectasis and IRI, may be reduced by TEAS, which is able to protect the lung from these types of injury. Further studies are necessary to explore the mechanism of action responsible for these processes.

\section{Acknowledgements}

The authors gratefully thank Dr Raymond Koehler from the Department of Anesthesiology and Critical Care Medicine, Johns Hopkins University (Baltimore, US) for his valuable input in the present study.

\section{Funding}

The present study was funded by the Ministry of Science and Technology of Liaoning Province, China (grant no. 20102282) and the Sanxin project of Shengjing Hospital of China Medical University (grant no. 2015PS57J) and the Support Plan for Innovative Talents in Liaoning Higher Education Institution (grant no. 201834).

\section{Availability of data and materials}

The datasets used and/or analyzed in the current study are available from the corresponding author on reasonable request.

\section{Authors' contributions}

WW, WB, YL and JZ designed the study. WW, WB, YY, XT, YL and YW performed of the study. WW, WB, XT and JZ performed the data analysis. WW, WB, YY and JZ prepared the manuscript. All authors contributed to the conception of the study and read and approved the final manuscript.

\section{Ethics approval and consent to participate}

Ethical approval of this study was granted by the Human Research Ethical Committee of Shengjing Hospital (Shenyang, China; IRB registration no. 2015PS57J). Informed consent was obtained from all of the participants.

\section{Patient consent for publication}

Not applicable.

\section{Competing interests}

The authors declare that they have no competing interests. 


\section{References}

1. Valenza F, Chevallard G, Fossali T, Salice V, Pizzocri M and Gattinoni L: Management of mechanical ventilation during laparoscopic surgery. Best Pract Res Clin Anaesthesiol 24: 227-241, 2010.

2. Strang CM, Hachenberg T, Fredén F and Hedenstierna G: Development of atelectasis and arterial to end-tidal $\mathrm{PCO} 2$-difference in a porcine model of pneumoperitoneum. $\mathrm{Br}$ J Anaesth 103: 298-303, 2009.

3. YP Deng and HS Huang: Effects of different pneumoperitoneal pressures on hemodynamics and arterial blood gas in geriatric patients during laparoscopic surgery. J Clin Anesthesiology 27: 741-743, 2011.

4. Simonson DA, Adams AB, Wright LA, Dries DJ, Hotchkiss JR and Marini JJ: Effects of ventilatory pattern on experimental lung injury cause by high airway pressure. Crit Care Med 32: 781-786, 2004.

5. PROVE Network Investigators for the Clinical Trial Network of the European Society of Anaesthesiology; Hemmes SN, Gama de Abreu M, Pelosi P and Schultz MJ: High versus low positive end-expiratory pressure during general anesthesia for open abdominal surgery (PROVHILO trial): A multicentre randomized controlled trial. Lancet 384: 495-503, 2014.

6. Fard N, Saffari A, Emami G, Hofer S, Kauczor HU and Mehrabi A: Acute respiratory distress syndrome induction by pulmonary ischemia-reperfusion injury in large animal models J Surg Res 189: 274-284, 2014.

7. Duggan $M$ and Kavanagh BP: Pulmonary atelectasis: A Pathogenic Perioperative Entity. Anesthesiology 102: 838-854, 2005.

8. Cinnella G, Grasso S, Spadaro S, Rauseo M, Mirabella L, Salatto P, De Capraris A, Nappi L, Greco P and Dambrosio M: Effects of recruitment maneuver and positive end-expiratory pressure on respiratory mechanics and transpulmonary pressure during laparoscopic surgery. Anesthesiology 118: 114-122, 2013.

9. Wolthuis EK, Choi G, Dessing MC, Bresser P, Lutter R, Dzoljic M, van der Poll T, Vroom MB, Hollmann M and Schultz MJ: Mechanical ventilation with lower tidal volumes and positive end-expiratory pressure prevents pulmonary inflammation in patients without preexisting lung injury. Anesthesiology 108: 46-54, 2008

10. Zhou F, Guo J, Cheng J, Wu G, Sun J and Xia Y: Electroacupuncture and brain protection against cerebral ischemia: Specific effects of acupoints. Evid Based Complement Alternat Med 2013: 804397 2013.

11. Daabiss M: American society of anaesthesiologists physical status classification. Indian J Anaesth 55: 111-115, 2011.

12. Arai YC, Kato N, Matsura M, Ito H, Kandatsu N, Kurokawa S, Mizutani M, Shibata Y and Komatsu T: Transcutaneous electrical nerve stimulation at the PC-5 and PC- 6 acupoints reduced the severity of hypotension after spinal anaesthesia in patients undergoing Caesarean section. Br J Anaesth 100: 78-81, 2008.

13. Ogna A, Nardi J, Prigent H, Quera Salva MA, Chaffaut C Lamothe L, Chevret S, Annane D, Orlikowski D and Lofaso F: Prognostic Value of initial assessment of residual hypoventilation Using nocturnal capnography in Mechanically Ventilated neuromuscular Patients: A 5-Year Follow-up study. Front Med (Lausanne) 3: 40, 2016.

14. Kim HJ, Lee J, Park YS, Lee CH, Lee SM, Yim JJ, Yoo CG, Kim YW, Han SK and Choi SM: Impact of GOLD groups of chronic pulmonary obstructive disease on surgical complications. Int J Chron Obstruct Pulmon Dis 11: 281-287, 2016.

15. Kim TH, Lee JS, Lee SW and Oh YM: Pulmonary complications after abdominal surgery in patients with mild-to-moderate chronic obstructive pulmonary disease. Int J Chron Obstruct Pulmon Dis 11: 2785-2796, 2016.

16. Biswas B, Sharma SK, Negi RS, Gupta N, Jaswal VM and Niranjan N: Pleural effusion: Role of pleural fluid cytology, adenosine deaminase level and pleural biopsy in diagnosis. J Cytol 33: 159-162, 2016.

17. MacDuff A, Arnold A and Harvey J: BTS Pleural Disease Guideline Group; Management of spontaneous pneumothorax: British thoracic society pleural disease guideline 2010. Thorax 65: ii18-ii31, 2010.

18. Maissen-Villiger CA, Schweighauser A, van Dorland HA, Morel C, Bruckmaier RM, Zurbriggen A and Francey T: Expression profile of cytokines and enzymes mRNA in blood leukocytes of dogs with leptospirosis and its associated pulmonary hemorrhage syndrome. PLoS One 11: e0148029, 2016.
19. Weng S, Wang L, Rong Y, Liu Y, Wang X, Guan H and Chen W: Effects of the interactions between dust exposure and genetic polymorphisms in nalp3, caspase-1 and IL-1 $\beta$ on the risk of silicosis: A case-control study. PLoS One 10: e0140952, 2015

20. Gielis JF, Jungraithmayr W, Boulet GA, Bogers JP, Weder W, Cos P and Van Schil PE: A murine model of lung ischemia and reperfusion injury: Tricks of the trade. J Surg Res 194: 659-666, 2015.

21. Ikeda K, Negishi H and Yamori Y: Antioxidant nutrients and hypoxia/ischemic brain injury in rodents. Toxicology 189: 55-61, 2003.

22. Strieter RM, Belperio JA and Keane MP: Cytokines in innate host defense in the lung. J Clin Invest 109: 699-705, 2002.

23. Funakoshi T, Ishibe Y, Okazaki N, Miura K, Liu R, Nagai S and Minami Y: Effect of re-expansion after short-period lung collapse on pulmonary capillary permeability and pro-inflammatory cytokine gene expression in isolated rabbit lungs. Br J Anaesth 92: 558-563, 2004.

24. Ashkenazi A and Dixit VM: Death receptors: Signaling and modulation. Science 281: 1305-1308, 1998.

25. Nagata S: Apoptosis by death factor. Cell 88: 355-365, 1997.

26. Okusawa S, Gelfand JA, Ikejima T, Connolly RJ and Dinarello CA: Interleukin 1 induces a shock-like state in rabbits. Synergism with tumor necrosis factor and the effect of cyclooxygenase inhibition. J Clin Invest 81: 1162-1172, 1988.

27. Ward PA: Role of complement, chemokines and regulatory cytokines in acute lung injury. Ann N Y Acad Sci 796: 104-112, 1996

28. Lalancette-Hébert M, Phaneuf D, Soucy G, Weng YC and Kriz J: Live imaging of toll-like receptor two response in cerebral ischemia reveals the role of olfactory bulb microglia as modulators of inflammation. Brain 132: 940-954, 2009.

29. Barton GM and Medzhitov R: Toll-like receptor signaling pathways. Science 300: 1524-1525, 2003.

30. Blanco AM, Pascual M, Valles SL and Guerri C: Ethanol-induced iNOS and COX-2 expression in cultured astrocytes via NF-kappa B. Neuroreport 15: 681-685, 2004.

31. Cannizzaro V, Hantos Z, Sly PD and Zosky GR: Linking lung function and inflammatory responses in ventilator-induced lung injury. Am J Physiol Lung Cell Mol Physiol 300: L112-L120, 2011.

32. Iqbal M, Multz AS, Rossoff LJ and Lackner RP: Reexpansion pulmonary edema after VATS successfully treated with continuous positive airway pressure. Ann Thorac Surg 70: 669-671, 2000.

33. Wake M, Sanagawa Y and Okamoto Y: A case of anesthetic management for reexpansion pulmonary edema of the dependent lung saved by superimposed HFJV during one-lung ventilation for the thoracoscopic operation associated with bilateral pneumothorax. Masui 49: 643-645, 2000 (In Japanese).

34. Yanagidate F, Dohi S, Hamaya Y and Tsujito T: Reexpansion pulmonary edema after thoracoscopic mediastinal tumor resection. Anesth Analg 92: 1416-1417, 2001.

35. Fang YG, Zhou XZ, Liu BY and Wang YY: A study on the essential drugs and points for point application in summer to treat the diseases with attacks in winter. J Tradit Chin Med 30: 180-184, 2010.

36. Carneiro ER, Carneiro CR, Castro MA, Yamamura Y and Silveira VL: Effect of electroacupuncture on bronchial asthma induced by ovalbumin in rats. J Altern Complement Med 11: $127-134,2005$.

37. Ngai SP and Jones AY: Changes in skin impedance and heart rate variability with application of Acu-TENS to BL 13 (Feishu). J Altern Complement Med 19: 558-563, 2013.

38. Li J, Wu S, Tang H, Huang W, Wang L, Zhou H, Zhou M, Wang H and Li J: Long-term effects of acupuncture treatment on airway smooth muscle in a rat model of the smoke-induced chronic obstructive pulmonary disease. Acupunct Med 34: 107-113, 2016.

39. Zhang Q, Gao Z, Wang H, Ma L, Guo F, Zhong H, Xiong L and Wang Q: The effect of pre-treatment with transcutaneous electrical acupoint stimulation on the quality of recovery after ambulatory breast surgery: A prospective, randomized controlled trial. Anaesthesia 69: 832-839, 2014.

40. Cai GF, Shang L, Liu K, Zhao H, Quan A, Yan C, Sun H, Li X and Zhuang Z: Remodeling of cross electro-nape-acupuncture on cough reflex in patients with a tracheotomy after cerebral hemorrhage: A randomized controlled trial. Zhongguo Zhen Jiu 35: 3-6, 2015 (In Chinese).

41. Fan WC, Ma W, Zhao C, Tong QY and Shen WD: Influence of acupuncture-drug compound anesthesia with different frequency electroacupuncture on immune function in patients undergoing pneumonectomy. Zhongguo Zhen Jiu 32: 715-719, 2012 (In Chinese). 
42. Fang XJ and Dong L: Acupuncture at Chize (LU5) for 23 cases of intractable hiccup. Zhongguo Zhen Jiu 35:1060, 2015 (In Chinese).

43. Chung JH, Lee EY, Jang MH, Kim CJ, Kim J, Ha E, Park HK, Choi S, Lee H, Park SH, et al: Acupuncture decreases ischemia-induced apoptosis and cell proliferation in the dentate gyrus of gerbils. Neurol Res 29 (Suppl 1): S23-S27, 2007.

44. Kang KA, Shin ES, Hur J, Hasan MR, Lee H, Park HJ, Park HK and Kim YJ: Acupuncture attenuates neuronal cell death in middle cerebral artery occlusion model of focal ischemia. Neurol Res 32 (Suppl 1): S84-S87, 2010.

45. Shen MH, Zhang CB, Zhang JH and Li PF: Electroacupuncture attenuates cerebral ischemia and reperfusion injury in middle cerebral artery occlusion of rat via modulation of apoptosis, inflammation, oxidative stress and excitotoxicity. Evid Based Complement Alternat Med 2016: 9438650, 2016.

46. Iadecola $\mathrm{C}$ and Alexander M: Cerebral ischemia and inflammation. Curr Opin Neurol 14: 89-94, 2001.

47. Parshall MB, Schwartzstein RM, Adams L, Banzett RB, Manning HL, Bourbeau J, Calverley PM, Gift AG, Harver A, Lareau SC, et al: An official American Thoracic Society statement: Update on the mechanisms, assessment and management of dyspnea. Am J Respir Crit Care Med 185: 435-452, 2012.
48. Bauml J, Haas A, Simone CB II, Li SQ, Cohen RB, Langer CJ and Mao JJ: Acupuncture for dyspnea in lung cancer: Results of a feasibility trial. Integr Cancer Ther 15: 326-332, 2016.

49. Jiang JH, Yang EJ, Baek MG, Kim SH, Lee SM and Choi SM: Anti-inflammatory effects of electroacupuncture in the respiratory system of asymptomatic amyotrophic lateral sclerosis animal model. Neurodegener Dis 8: 504-514, 2011.

50. Gu G, Zhang Z, Wang G, Han F, Han L, Wang K, Liu J and Li W: Effects of electroacupuncture pretreatment on inflammatory response and acute kidney injury in endotoxemic rats. J Int Med Res 39: 1783-1797, 2011.

51. Lan L, Tao J, Chen A, Xie G, Huang J, Lin J, Peng J and Chen L: Electroacupuncture exerts anti-inflammatory effects in cerebral ischemia-reperfusion injured rats via suppression of the TLR4/NF-кB pathway. Int J Mol Med 31: 75-80, 2013.

This work is licensed under a Creative Commons Attribution-NonCommercial-NoDerivatives 4.0 International (CC BY-NC-ND 4.0) License. 